P035 THE TRANSCRIPTIONAL CO-ACTIVATOR BOB.1 PREVENTS TERMINAL DIFFERENTIATION AND INDUCES COSTIMULATORY CAPACITY OF B CELLS IN GC-LIKE ENVIRONMENT

1,2,3 MU Levels, 1,2,3 CM Fehres, 1,2,3 NOP Van Uden, ${ }^{4}$ AQ Bakker, 4,5 H Spits, 1,2,3 DL Baeten,

${ }^{1,2,3} \mathrm{NG}$ Yeremenko*. ${ }^{1}$ Amsterdam Rheumatology and immunology Centre; ${ }^{2}$ Clinical Immunology and Rheumatology; ${ }^{3}$ Experimental Immunology, Academic Medical Centre/ University of Amsterdam, Academic Medical Centre/University of Amsterdam; ${ }^{4}$ AIMM Therapeutics; ${ }^{5}$ Department of Cell Biology and Histology, Academic Medical Centre/ University of Amsterdam, Amsterdam, Netherlands

\subsection{6/annrheumdis-2018-EWRR2018.57}

Introduction Recently we identified the transcriptional co-activator BOB.1 as specifically overexpressed in RA synovium, where its levels strongly correlated with the presence of germinal centres (GCs). In accordance with human data, mice lacking functional BOB.1 failed to mount GC response and were resistant to the experimental model of RA.

Objectives In this study we investigated whether increased levels of BOB.1 impact the phenotype and function of B cells in a GC-like environment.

Methods Expression levels of BOB.1 were assessed by qPCR and immunofluorescence. DNA encoding BOB.1 was introduced into B cells by retroviral-mediated gene transfer. The phenotype, activation and BCR signalling were analysed by FACS. Expression of CCL3 was measured by qPCR. Impact of high levels of BOB.1 in B cells on proliferative responses of autologous $\mathrm{T}_{\mathrm{FH}}$ cells was assessed by FACS after 5 days of co-culture with SEB-pulsed B cells.

Results Immunofluorescence staining of inflamed RA synovium demonstrated the presence of high numbers of BOB.1-positive cells clustered in the lymphoid follicles in a pattern similar to the normal tonsillar tissue. Further analysis revealed that expression of BOB.1 in RA synovial B cells was significantly higher than in tonsillar B cells. To investigate how high levels of BOB.1 impact phenotype and function of B cells in a GClike environment we overexpressed BOB.1 in human primary B cells cultured with CD40L and IL-21. In these conditions B cells rapidly differentiate into antibody-producing plasma cells, a process that is accompanied by a decrease in expression of CD20 and increase in CD38 and CD27. In contrast to the control, cells transduced with BOB.1 retained CD20 expression while expressed intermediate levels of CD27 and CD38. Accordingly, the percentage of plasmablasts was significantly lower in BOB.1-overexpressing cells, confirming that high levels of BOB.1 suppress plasma cell differentiation. Further analysis revealed that BOB.1-transduced memory B cells expressed high levels of costimulatory receptors CD40, CD80 and PD$\mathrm{L} 2$ involved in B-T cell interactions and showed more rapid $\mathrm{Ca}^{2+}$ mobilisation and increased production of T-cell-recruiting chemokine CCL3 following BCR stimulation. Accordingly, $\mathrm{T}_{\mathrm{FH}}$ cells co-cultured with BOB.1-transduced B cells exhibit a higher rate of proliferation as compared to $\mathrm{T}_{\mathrm{FH}}$ cells co-cultured with $\mathrm{B}$ cells transduced with the control retrovirus.

Conclusions These data suggest that increased levels of BOB.1 in B cells during $\mathrm{T}$ cell-dependent responses in GCs suppress their terminal differentiation and enhance expression of costimulatory molecules and BCR signalling strength, which is translated into their greater help to $\mathrm{T}_{\mathrm{FH}}$ cells. Whether this is sufficient to drive/accelerate autoimmune disease will be evaluated in a B cell-specific BOB.1 tg mouse model.

Disclosure of interest None declared

\section{P036 NEUTROPHIL EXTRACELLULAR TRAPS IN SYSTEMIC LUPUS: A PROTEOMIC ANALYSIS}

${ }^{1} \mathrm{~F}$ Pratesi, ${ }^{2} \mathrm{M}$ Bruschi, ${ }^{2} \mathrm{~A}$ Bonanni, ${ }^{2} \mathrm{~A}$ Petretto, ${ }^{1} \mathrm{I}$ Puxeddu, ${ }^{2} \mathrm{G}$ Candiano, ${ }^{2} \mathrm{G}$ Ghiggeri, ${ }^{1} \mathrm{P}$ Migliorini*. ${ }^{1}$ Clinical and Experimental Medicine, University of Pisa, Pisa; ${ }^{2}$ Division of Nephrology, Dialysis and Transplantation, Istituto G. Gaslini, Genova, Italy

\subsection{6/annrheumdis-2018-EWRR2018.58}

Introduction An altered formation and/or removal of neutrophil extracellular traps (NET) has been recently proposed as key event in systemic lupus (SLE): in fact, NETs have been detected in the skin and kidney of SLE patients, low density granulocytes from SLE patients form more frequently NETs and NET degradation is impaired. However, no data are yet available on NET composition in SLE patients.

Objectives The aim of the study is to compare NETs from SLE patients and normals by means of proteomic analysis.

Methods Neutrophils from 11 normals and 16 SLE patients (9/16 with active disease) were stimulated by PMA, NET proteins were released in supernatants by DNase treatment and characterised by Fusion Orbitrap Mass spectrometry.

Results Overall, 802 proteins were detected (50\% identified in all the samples); 236 are exclusively found in control NETs, 57 only in active SLE and 22 in inactive SLE. Multidimensional scaling (MDS) showed that NETs composition/amount allows the identification of 3 different clusters corresponding to active SLE, inactive SLE and controls in a bi-dimensional scatter plot. The heat map describing the proteome profiles shows two different clusters of proteins, one represented by 16 proteins that were more expressed in inactive disease, the other by 9 proteins more expressed in active SLE. A preliminary analysis of posttranslational modifications showed a differential expression of methyloxidized and phosphorylated proteins.

Conclusions The data obtained suggest that NET composition and type/extent of posttranslational modifications are different in active SLE, in inactive disease and in controls. The different cytokine and autoantibody environment that distinguishes SLE flares from inactive may activate different pathways in NET induction and condition the quality and quantity of NET-associated proteins.

Disclosure of interest None declared

\section{P037 MULTIPLE ROLES OF PHOSPHOLIPASE C-ETA2, AS A NOVEL C2 DOMAIN CONTAINING PROTEIN, IN THE PATHOGENESIS OF RHEUMATOID ARTHRITIS}

${ }^{1}$ SI Lee*, ${ }^{1} \mathrm{JH}$ Park, ${ }^{1} \mathrm{HS}$ Noh, ${ }^{2}$ W-U Kim. 'Internal Medicine, Gyeongsang National University School of Medicine, Jinju; ${ }^{2}$ Internal Medicine, the Catholic University of Korea, Seoul, Korea, Republic of Ireland

\subsection{6/annrheumdis-2018-EWRR2018.59}

Introduction The $\mathrm{C} 2$ domain is a $\mathrm{Ca}^{2+}$-dependent membrane-targeting motif found in many cellular proteins involved in signal transduction or inflammation pathway. However, the effects of C2 domain-containing proteins in the fibroblast-like synoviocyte (FLS) of rheumatoid arthritis (RA) have not yet been elucidated.

Objectives The aim of this study was to screen novel C2 domain-containing proteins related to aggressiveness of FLS, and confirm the precise roles of target protein in RA.

Methods We transduced RA-FLS with a recombinant adenovirus expressing a C2 domain library. To confirm the effect of phospholipase C-eta2 (PLCH2), as a candidate C2 domain- 
containing target protein, we established stable MH7A cell lines overexpressing GFP-tagged PLCH2. We also conducted in vitro and in vivo experiments using a recombinant adenovirus expressing the full length form of $\mathrm{PLCH} 2$ or $\mathrm{C} 2$ domain of PLCH2 (C2-PLCH2).

Results Several C2 domain-containing proteins were identified whose over-expression resulted in reduced proliferation and NF-kB activity of RA-FLS. Among those, we focused on $\mathrm{PLCH} 2$ in this study. PLCH2 levels were significantly decreased in RA-FLS and synovium than OA. PLCH2 and C2$\mathrm{PLCH} 2$ suppressed cell invasion and migration, cytokines production, and matrix metalloproteinase secretion in RA-FLS and stable MH7A cell lines compared to control. PLCH2 and C2PLCH2 sensitised RA-FLS to apoptosis in vitro and in vivo model of matrigel implants engrafted into immunodeficient mice. PLCH2 and C2-PLCH2 further decreased arthritis severity in collagen-induced arthritis (CIA) mouse model.

Conclusions Our results showed that invasive characteristics of RA-FLS and inflammatory arthritis of CIA are reduced by $\mathrm{PLCH} 2$ and $\mathrm{C} 2$ domain of PLCH2. Therefore C2 domain of $\mathrm{PLCH} 2$ may have therapeutic potential for RA.

\section{REFERENCES}

1. Farah $C A$, Sossin WS. The role of $C 2$ domains in PKC signalling. Adv Exp Med Biol 2012; 740:663-83.

2. Yáñez M, Gil-Longo J, Campos-Toimil M. Calcium binding proteins. Adv Exp Med Biol 2012;740:461-82.

3. Friedrich $R$, Yeheskel $A$, Ashery U. DOC2B, C2 domains, and calcium: $A$ tale of intricate interactions. Mol Neurobiol 2010 February;41(1):42-51.

4. Zhou Y, Sondek J, Harden TK. Activation of human phospholipase C-eta2 by Gbetagamma. Biochemistry 2008 April 15;47(15):4410-7.

5. Popovics P, Beswick W, Guild SB, Cramb G, Morgan K, Millar RP, Stewart AJ. Phospholipase C $-\eta 2$ is activated by elevated intracellular $\mathrm{Ca}(2+)$ levels. Cell Signal 2011 November;23(11):1777-84.

Acknowledgements This work was supported by grants (NRF2014R1A2A1A11051360 and NRF-2015R1A5A2008833) from the National Research Foundation of Korea (NRF) funded by the Korean government (MSIP).

Disclosure of interest None declared

\section{P038 RECENT THYMIC EMIGRANT T CELLS INCREASE IN RHEUMATOID PATIENTS TREATED WITH ABATACEPT}

\footnotetext{
${ }^{1,2} \mathrm{~S}$ Piantoni* ${ }^{*}{ }^{1} \mathrm{~F}$ Regola, ${ }^{1} \mathrm{~A}$ Tincani, ${ }^{1} \mathrm{P}$ Airò. ${ }^{1}$ Rheumatology and Clinical Immunology Unit, Spedali Civili and University of Brescia; ${ }^{2}$ Department of Molecular and Translational Medicine, University of Brescia, Brescia, Italy
}

\subsection{6/annrheumdis-2018-EWRR2018.60}

Introduction CD4 $+\mathrm{T}$ cells in rheumatoid arthritis (RA) display a peculiar restriction of the $T$-cell receptor (TCR) repertoire which compromises their ability to react to novel antigens. ${ }^{1}$ We demonstrated that this process could be partially reverted by abatacept (ABA), which is a blocker of T lymphocyte costimulation, used in the treatment of RA. ${ }^{2}$

To better understand the mechanisms underlying this phenomenon, we speculated that ABA could influence the frequency of peripheral $\mathrm{T}$ cell subpopulations, even at early stages of maturation.

The homeostasis of the naive T cell pool is maintained not only by thymus production, but also by mechanisms of peripheral replication involving TCR activation revealed by CD31 down-modulation. Indeed, CD4+CD45RA + CD31+T cells have been described as recent thymic emigrants (RTE) newly produced by the thymus and CD4+CD45RA+CD31 T cells as central naïve, involved in a self-renewal peripheral process.

Objectives We aimed at profiling circulating naïve CD4 + CD45RA+T cells, by assessing their expression of CD31 (PECAM-1). ${ }^{3}$

Methods Thirty-one RA patients (median age $\left[10^{\circ}-90^{\circ}\right.$ percentile] 42 [25-64] years) were evaluated before and after 6 month of ABA therapy. Phenotypic analysis of peripheral blood $\mathrm{T}$ lymphocytes was made by flow-cytometry. Wilcoxonsigned rank test and Spearman rank correlation were used for the comparisons.

Results After ABA therapy, the absolute number of total $\mathrm{T}$ CD4 +increased from 780 [423-1351] to 1000 [658-1566] cells $/ \mathrm{mm}^{3}(\mathrm{p}=0.01)$. Total naive CD4 +increased in percentage (33 [18-56] vs $40[20-61] \%$ of CD4+; $p=0.02)$ and in absolute number $\left(257\right.$ [82-568] vs 344 [62-689] cells $/ \mathrm{mm}^{3}$; $\mathrm{p}=0.03)$. In parallel, the number of RTE increased in percentage $(10.6[2-26]$ vs $11.6[3-25] \%$ of $C D 4+; p=0.04)$ and in absolute number (51 [15-194] vs 110 [23-271] cells $/ \mathrm{mm}^{3}$; $\mathrm{p}=0.01)$. The central naïve counterpart did not show significant variations in percentage $(29[23-40]$ vs $27[20-38] \%$ of CD4+; $\mathrm{p}=0.20$ ) nor in absolute number (191 [41-404] vs 242 [76-526] cells $\left./ \mathrm{mm}^{3} ; \mathrm{p}=0.20\right)$. No correlation was found between age and RTE number at baseline.

Conclusions The number of total naive T cells increases after therapy with $\mathrm{ABA}$ together with the number of RTE, suggesting a thymic output boost. Besides the peripheral effect in reducing the number of effector T cells which was showed by previous studies, ${ }^{4}$ ABA could have a role in promoting the immune reconstitution at the early stage of T cell development.

\section{REFERENCES}

1. Wagner UG, et al. PNAS 1998.

2. Imberti L, et al. J Trans/ Med 2015.

3. Kohler $\mathrm{S}$, et al. Blood 2009.

4. Airò P, et al. Trends Mol Med 2013.

Disclosure of interest None declared

\section{P039 ROTATOR CUFF CALCIFIC TENDINOPATHY: CHONDROCYTE-LIKE CELLS SURROUNDING CALCIFIC DEPOSITS EXPRESS TNAP AND ENPP1, TWO KEY ENZYMES OF THE MINERALIZATION PROCESS}

${ }^{1,2} \mathrm{C}$ Darrieutort-Laffite*, ${ }^{1,2} \mathrm{~A}$ Najm, ${ }^{2} \mathrm{~T}$ Garraud, ${ }^{2} \mathrm{P}$ Layrolle, ${ }^{2} \mathrm{~F}$ Blanchard, $1,2 \mathrm{~B}$ Le Goff.
${ }^{1}$ Rheumatology, CHU de Nantes; ${ }^{2}$ INSERM U1238, Phy-Os Laboratory, Nantes, France

10.1136/annrheumdis-2018-EWRR2018.61

Introduction Calcific tendinopathy is a frequent cause of chronic painful shoulders. It is caused by deposits of carbonated apatite in rotator cuff tendons. Although the disease is frequent, molecular and cellular mechanisms involved in this pathological mineralization process are not currently identified. Objectives The objective of the study was to analyse calcified tendon samples to understand the organisation of the deposits and to characterise the cells potentially involved in their formation.

Methods Samples were collected from cadaveric subjects. They were fixed in formalin 4\%, decalcified in EDTA, dehydrated and embedded in paraffin. Some samples were not decalcified to allow a better characterisation of the calcific deposits. Hematoxylin and eosin (HE), Safranin O/Fast Green (SO/FG), Von Kossa (no decalcified samples) and Tartrate-Resistant Acid Phosphatase (TRAP) 